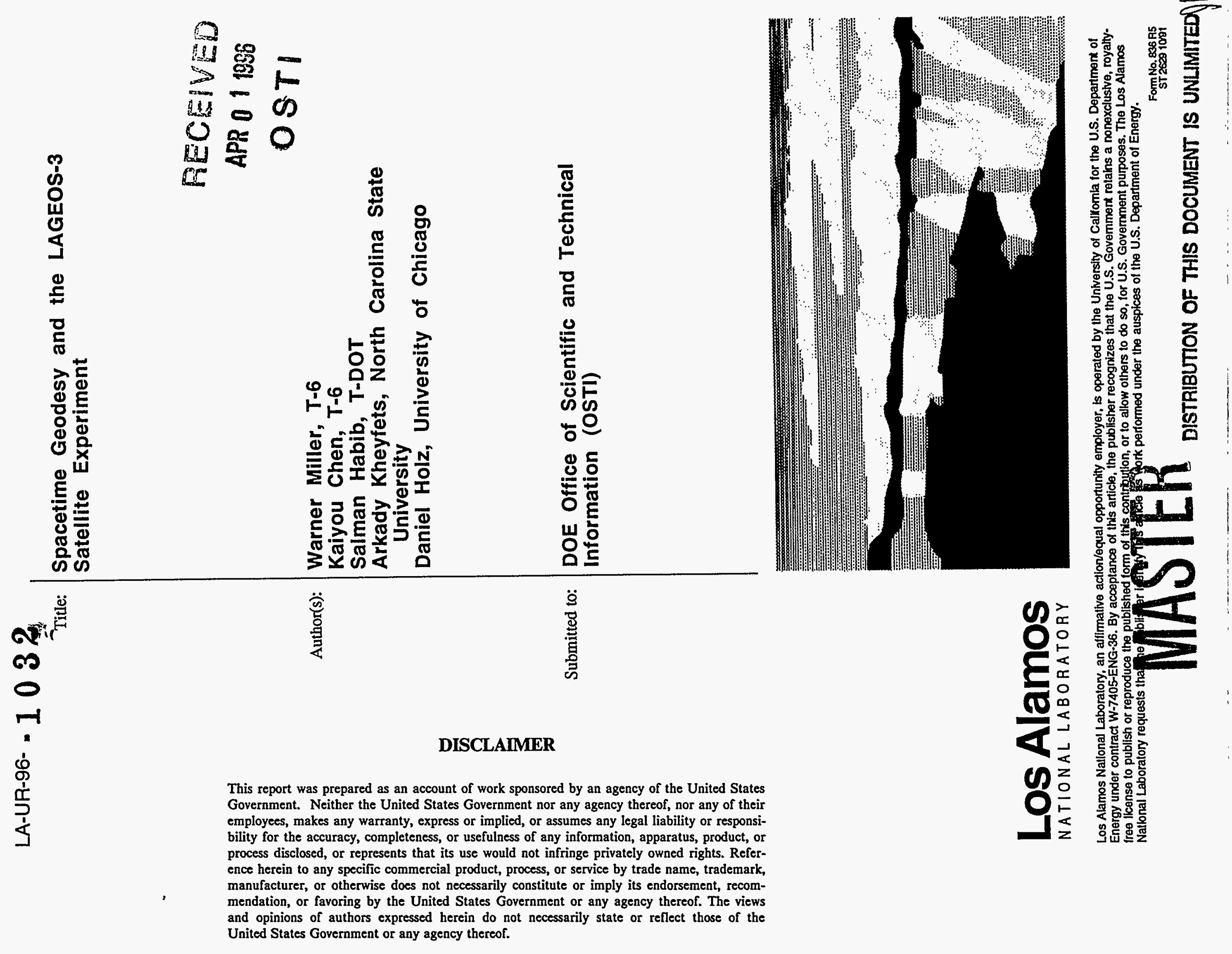




\title{
Spacetime Geodesy and the LAGEOS-3 Satellite Experiment
}

\author{
Warner A. Miller*, Kaiyou Chen, Salman Habib, Arkady Kheyfets \\ (North Carolina State University), and Daniel E. Holz (University of Chicago)
}

\begin{abstract}
This is the final report of a three-year, Laboratory-Directed Research and Development (LDRD) project at the Los Alamos National Laboratory (LANL). LAGEOS-1 is a dense spherical satellite whose tracking accuracy is such as to yield a medium-term inertial reference frame and that is used as an adjunct to more difficult and more data-intensive absolute frame measurements. LAGEOS-3, an identical satellite to be launched into an orbit complementary to that of LAGEOS-1, would experience an equal and opposite classical precession to that of LAGEOS-1. Besides providing a more accurate real-time measurement of the earth's length of day and polar wobble, this paired-satellite system would provide the first direct measurement of the general relativistic frame-dragging effect. Of the five dominant error sources in this experiment, the largest one involves surface forces on the satellite and their consequent impact on the orbital nodal precession. The surface forces are a function of the spin dynamics of the satellite. We have modeled the spin dynamics of a LAGEOS-type satellite and used this spin model to estimate the impact of the thermal rocketing effect on the LAGEOS-3 experiment. We have also performed an analytic tensor expansion of Synge's world function to better reveal the nature of the predicted frame-dragging effect. We showed that this effect is not due to the Riemann curvature tensor, but rather is a "potential effect" arising from the acceleration of the world lines in the Kerr spacetime geometry.
\end{abstract}

\section{Background and Research Objectives}

The proposed Laser Geodynamic Satellite Experiment-3 (LAGEOS-3) would measure, for the first time, a new quasi-stationary property of the earth -- its gravitational magnetic

*Principal investigator, e-mail: wam@lanl.gov 
dipole moment (gravitomagnetism) predicted by Einstein's theory of general relativity. This gravitomagnetic field causes the local inertial frames to be dragged around with the earth at a rate proportional to the angular momentum of the earth and inversely proportional to the cube of the distance from the center of the earth. This will cause the line of nodes of the orbital plane of LAGEOS-3 to precess eastward at $32 \mathrm{mas} / \mathrm{yr}$. Although, in this example, the frame-dragging effect is small compared to the torque on the orbital plane due to the oblateness of the earth, it is an essential ingredient in the dynamics of accretion disks, binary systems, and other astrophysical phenomena.

The focus of our research was twofold: (1) to develop a spin model of LAGEOS to determine the expected thermal rocketing induced error in the experiment, thereby reducing the largest source of error, and (2) to explore further the nature of the frame-dragging effect and determine if its effect can be observed in an astrophysics setting.

\section{Importance to LANL's Science and Technology Base and National R\&D Needs}

Today, almost eighty years after Einstein introduced his geometric theory of gravity, we have just begun to measure - to verify - his gravitation theory. Of no less stature than the "tide producing" $-\mathrm{M} / \mathrm{r}^{2}$ "electric component" of gravity is the inertial-frame-defining "magnetic component" of gravitation $-\mathrm{J} / \mathrm{r}^{3}$. To see this force in action, first inject a satellite into a polar orbit about an earth-like mass idealized as not spinning with respect to the distant quasars. The satellite will remain in orbit in a continuous acceleration toward the center-of-mass of the attracting body under the influence of the Newtonian $1 / \mathrm{r}^{2}$ force, and its orbital plane will remain fixed in orientation with respect to distant quasars. Second, spin this central body, give it angular momentum and follow the trajectory of the satellite. Its orbital plane will experience a torque along the body's rotation axis. The orbital plane will undergo a precessional motion in the direction of the central body's rotation. The mass in motion of the central body or "mass current" produces a dipole gravitational field - the gravitomagnetic field. In the case of a satellite orbiting at two earth radii, the orbital plane will precess about the body axis of the earth at approximately $32 \mathrm{mas} / \mathrm{yr}$. This is the Lense-Thirring effect.

This force has never been directly measured. A measurement of this gravitomagnetic force is arguably comparable to the pioneering work of Michael Faraday on the measurement of a magnetic force between two current-carrying wires. However, the laboratory setting for this gravity measurement will be the 4-dimensional curved spacetime (approximately Kerr) geometry enveloping the earth. The idea behind this gravity measurement is simple. Whereas the EverittFairbanks experiment (Standford Gravity Probe-B) proposes a polar orbiting gyroscope, the 
Ciufolini LAGEOS-3/LAGEOS-1 experiment proposes to use the orbital plane itself as a gyroscope.

The spin dynamics modeling and subsequent analysis of the nodal precession of the LAGEOS orbital plane will diminish the largest error source in the LAGEOS-3 experiment. The accuracy of the Lense-Thirring effect can then be increased a factor of two, reducing the experimental error to a 2 percent level.

\section{Scientific Approach and Results}

To circumvent the need for expensive satellite experiments (e.g. Stanford Gravity Probe B experiment and the LAGEOS-3 experiment), we have undertaken an analysis of the Doppler broading of Fe lines from an accretion disk around a spinning black hole. If we can measure the angular momentum of a black hole astronomically, then this would alleviate the need for the satellite experiments. We will continue to determine the effect on the Fe line profiles from the angular momentum of the black hole. Recent observational results from a Japanese $x$-ray satellite indicates that there is a real possibility that such a measurement can be performed in the near future.

We modeled the spin dynamics of a LAGEOS-type satellite and used this spin model to estimate the impact of the thermal rocketing effect on the LAGEOS-3 experiment (Fig. 1). We also answered the following four questions that arose from the spin modeling:

(1) Can we obtain the asymptotic solution analytically and in so doing can we understand the wobbling or slippage of the Euler angles with respect to the relatively stable total instantaneous angular velocity?

(2) Can we understand why the rms fluctuations in the gravitational potential energy cause the asymptotic value of the angular momentum vector to be offset from the orbital plane by $\sim 10$ degrees?

(3) Can we understand why the nutation angle ( $\theta)$ drifts initially at a rate of $-20 \mathrm{deg} / \mathrm{yr}$ and reaches a pseudo-stable value of $\sim 115 \mathrm{deg}$ ?

(4) Can we understand the fluctuations in the spin rate $(\psi)$ over the first -20 yrs, which do not appear to have been detected experimentally?

We performed the tensor expansion of Synge's world function and proved that the framedragging effect arises from the connection coefficient terms rather than from the Riemann curvature tensor. Finally, we calculated the Doppler shift of Fe lines emitted from an accretion disk around a spinning black hole. We have determined the dependency of the Doppler shift on the angular momentum of the black hole using a numerical code we developed. Figure 2 shows a contour image generated by our code for such an accretion disk. 


\section{Publications}

[1] S. Habib, D. Holz, A. Kheyfets, R. Matzner, W. Miller and B. Tolman, "Spin Dynamics of the LAGEOS Satellite in Support of a Measurement of the Earth's Gravitomagnetism," Phys. Rev., D50, 6068 (1994).

[2] D. Holz, K. Chen and W. Miller, "Can We Measure the Angular Momentum of a Black Hole?," submitted to Phys. Rev. Lett. (1995). 

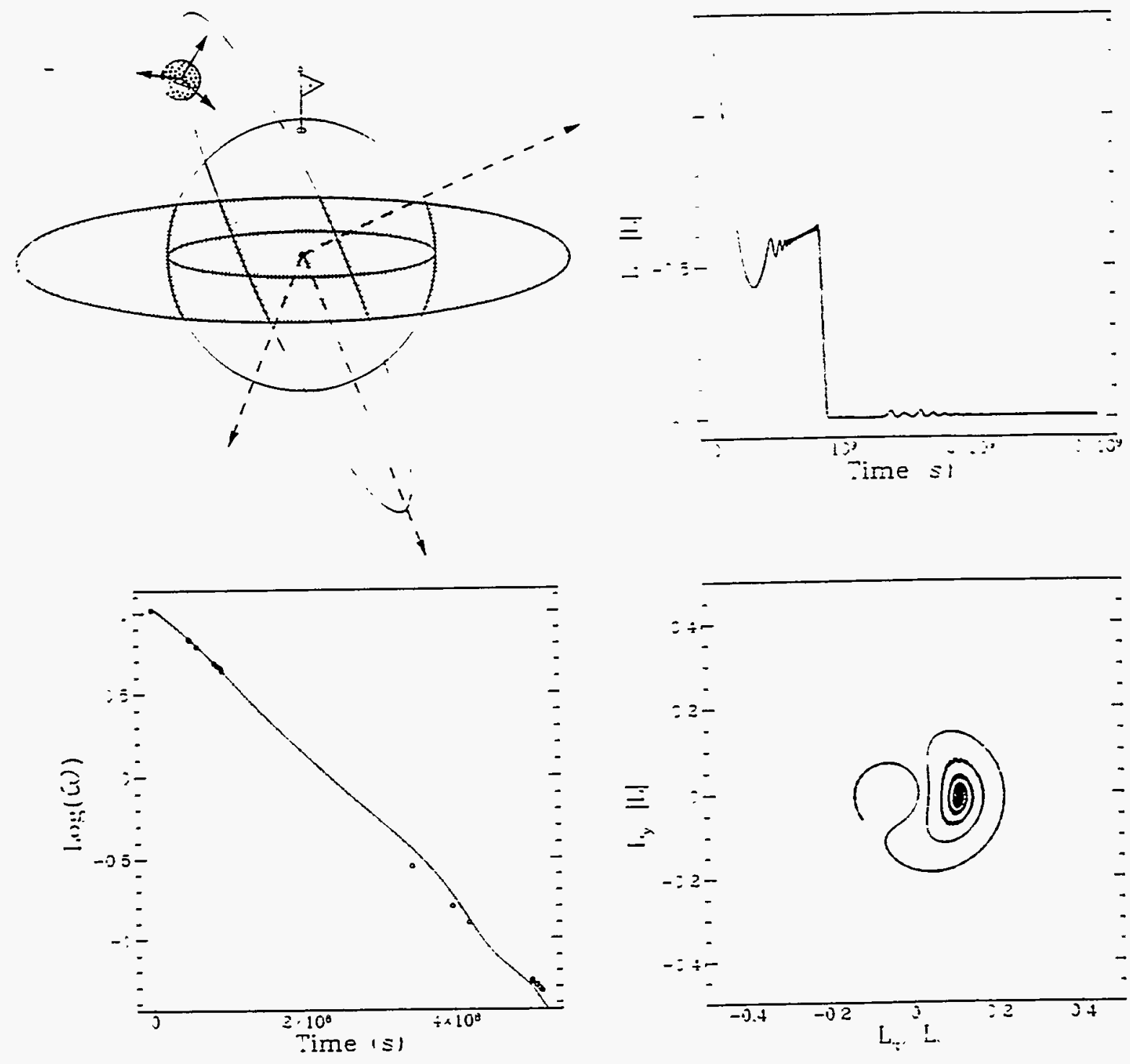

Fig. 1. Dynamics of the Euler angles. The evolution of the nutation (angle of obliquity, $\theta$ ) of the model satellite (upper left). In the asymptotic limit the angular velocities of nutation and precession average to zero (upper and lower right, respectively). However, the precession rate $\omega_{\phi}$ locks into the orbital velocity. Into the asymptotic limit (lower left): this plot demonstrates clearly the dynamics through the spin-orbit resonance phase. 


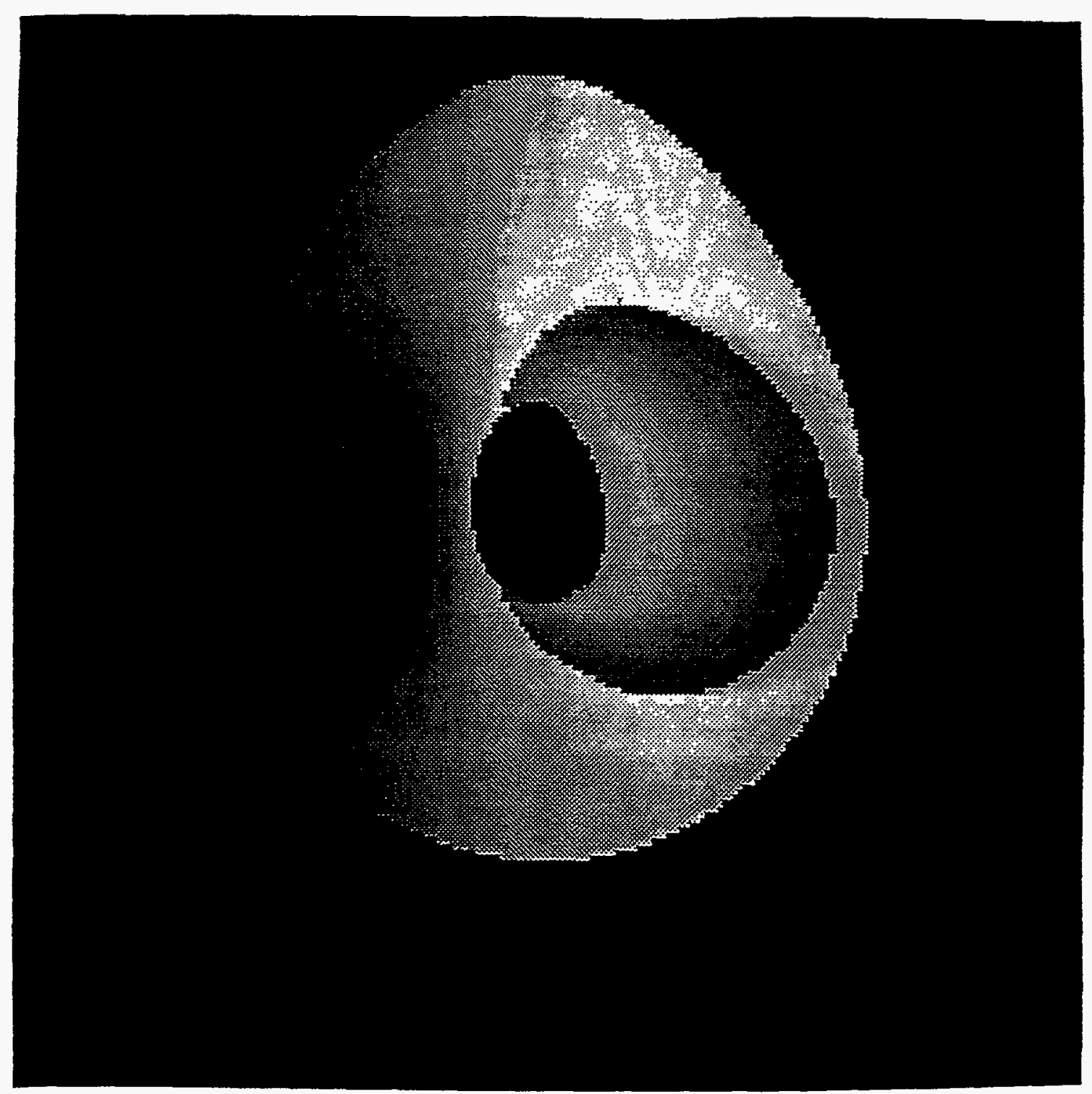

Fig. 2. Contour plot of a thin accretion disk about a maximally spinning black hole of mass $M$. The inner radius of the disk is at $3 \mathrm{M}$ while the outer edge is at 20M. The distortion was calculated using a general relativistic ray-tracing code developed under this project. The inclination of the photographic plate is at $60 \mathrm{deg}$ (the most probable viewing angle), while the distance from the black hole was $1000 \mathrm{M}$. The distortion of the disk is caused by the bending of the light rays by the spinning black hole. This is why the disk appears oblong in the "wrong" direction. The contours represent the observed Dopplershifted frequencies of the Fe lines. 